\title{
SUFFICIENT CONDITIONS FOR THE INTERSECTION PROPERTY IN GENERALIZED LIÉNARD SYSTEMS
}

\author{
YONG-IN KIM
}

Abstract. Some new results on the intersection property of all nonzero solutions of a class of planar systems of Liénard type with vertical isoclines are obtained. The results of this paper generalize some previous results on this field.

\section{INTRODUCTION}

In this paper, we consider the intersection property of all solutions of the following generalized Liénard systems with vertical isoclines:

$$
\left\{\begin{array}{l}
\phi_{p}\left(x^{\prime}\right)=\frac{y-F(x)}{a(x)}, \\
y^{\prime}=-a(x) g(x),
\end{array}\right.
$$

where $^{\prime}=d / d t, \phi_{p}(u)=|u|^{p-2} u, p>1$ and

$$
\left\{\begin{array}{l}
x^{\prime}=\frac{h(y)-F(x)}{a(x)}, \\
y^{\prime}=-a(x) g(x),
\end{array}\right.
$$

with $h(y)=m|y|^{k-1} y, m>0$ and $k \geq 1$. We assume throughout this paper that $F, a, g$ are continuous functions on $\mathbb{R}$ such that $F(0)=g(0)=0, x g(x)>0$ for all $x \neq 0$ and $a(x)>0$ for all $x \in \mathbb{R}$. Moreover, we assume that smoothness conditions for the existence and uniqueness of solutions for the initial value problem of (1) and (2) are satisfied. The following second order $p$-Laplacian:

$$
\left(\phi_{p}\left(x^{\prime}\right)\right)^{\prime}+f(x) x^{\prime}+g(x)=0,
$$

can be written as system (1) if we put $a(x)=1, F(x)=\int_{0}^{x} f(s) d s$ and $y=\phi_{p}\left(x^{\prime}\right)+$ $F(x)$. In particular, if $a(x)=1, p=2$, then system (1) reduces to the so-called

Received by the editors March 29, 2011. Revised July 17, 2011. Accepted August 8, 2011. 2000 Mathematics Subject Classification. 34A12, 34A34.

Key words and phrases. generalized Liénard system, $p$-Laplacian, vertical isocline. 
Liénard system

$$
\left\{\begin{array}{l}
x^{\prime}=y-F(x), \\
y^{\prime}=-g(x),
\end{array}\right.
$$

which is equivalent to the following second order equation

$$
x^{\prime \prime}+f(x) x^{\prime}+g(x)=0 .
$$

Let us consider system (1) or (2). It is easy to see that the origin is the unique equilibrium and the vector field (1) or (2) must pass through the positive $y$-axis from left to right and the positive $x$-axis from above to below and the negative $y$ axis from right to left and the negative $x$-axis from below to above. Hence, in order to study the global asymptotic stability of the zero solution, oscillation problem and existence of periodic solutions of (1) or (2), it is important to find out the conditions to decide whether all orbits of (1) or (2) intersect the vertical isocline $y=F(x)$ or $h(y)=F(x)$. Indeed, this property is a crucial step for the proof of the existence of periodic orbits and oscillation of all solutions of (1) or (2). We first recall some previous results on this topic and some definitions. We say that system (1) or (2) has property $X^{+}$if each positive orbit of (1) or (2) starting from $\left(0, y_{0}\right)$ with $y_{0}>0$ crosses the vertical isocline $y=F(x)$ or $h(y)=F(x)$ with some $x>0$ in finite time. We say that system (1) or (2) has property $X^{-}$if each positive orbit starting from $\left(0, y_{0}\right)$ with $y_{0}<0$ crosses the vertical isocline $y=F(x)$ or $h(y)=F(x)$ for some $x<0$. If system (1) or (2) has both property $X^{+}$and property $X^{-}$, we say that it has property $X$. Many results have been achieved on this topic for system (4) and its equivalent form (5), see, for example, [1-11,13-15] and the references therein. Recently, Sugie et al [15] have obtained some explicit conditions for system (2) (with $a(x)=1$ ) to have property $X^{+}$and Hesaaraki et al [13] also have obtained some implicit conditions for system (2) to have property $X^{+}$. Some previous results gave conditions on $f$ to discuss the property $X^{+}$. Moreover, Aghajani et al [12] have obtained some new explicit sufficient conditions for system (4) to have property $X^{+}$. The main results of [12] are the following theorems:

Let

$$
G(x)=\int_{0}^{x} g(s) d s, \quad \frac{d s}{d t}=\frac{|g(x)|}{\sqrt{2 G(x)}}, \quad u=\sqrt{2 G(x)} \operatorname{sgn}(x) .
$$


Then system (4) is equivalent to the following canonical form of Liénard system:

$$
\left\{\begin{array}{l}
\frac{d u}{d s}=y-F^{*}(u) \\
\frac{d y}{d s}=-u
\end{array}\right.
$$

where $F^{*}(u)=F\left(G^{-1}\left(u^{2} / 2\right)\right)$ if $u \geq 0$ and $F^{*}(u)=F\left(G^{-1}\left(-u^{2} / 2\right)\right)$ if $u<0$ and $G^{-1}$ is the inverse function of $G(x) \operatorname{sgn}(x)$.

Theorem A. Suppose that for some $b>0$,

$$
\limsup _{u \rightarrow+\infty}\left(\int_{b}^{u} \frac{F^{*}(s)+2 s}{s^{2}} d s+\frac{F^{*}(u)}{u}\right)=+\infty .
$$

Then system (6) has property $X^{+}$in the right half-plane.

Theorem B. Assume that $G(+\infty)=+\infty$ and that for some $b>0$,

$$
\limsup _{x \rightarrow+\infty}\left(\int_{b}^{x}\left(\frac{F(s) g(s)}{(2 G(s))^{\frac{3}{2}}}+\frac{g(s)}{G(s)}\right) d s+\frac{F(x)}{\sqrt{2 G(x)}}\right)=+\infty .
$$

Then system (4) has property $X^{+}$in the right half-plane.

In this paper, we generalize the results of [12] to system (1) and (2). Moreover, our results are different from those of [15] when $a(x)=1$.

\section{Coordinates Transformations And Lemmas}

First, by using coordinates and time transformations, we change system (1) and (2) into the so called canonical forms. Let $q=\frac{p}{p-1}$. Then $q>1$ and $\frac{1}{p}+\frac{1}{q}=1$.

Lemma 1. Define coordinates and time transformation $T_{1}$ as

$$
T_{1}: u=(p \bar{G}(x))^{\frac{1}{p}} \operatorname{sgn} x, \quad y=y, \quad \frac{d s}{d t}=\frac{a(x)|g(x)|}{(p \bar{G}(x))^{\frac{1}{q}}},
$$

where $\bar{G}(x)=\int_{0}^{x} a^{q}(s) g(s) d s$. Then, under the transformation $T_{1}$, system (1) is changed into the following form:

$$
\left\{\begin{array}{l}
\phi_{p}(\dot{u})=y-F^{*}(u) \\
\dot{y}=-\phi_{p}(u)
\end{array}\right.
$$

where $\dot{u}=\frac{d u}{d s}, \dot{y}=\frac{d y}{d s}$ and

$$
F^{*}(u)=F\left(\bar{G}^{-1}\left(|u|^{p} / p\right)\right)
$$

with $\bar{G}^{-1}>0$ if $u>0$ and $\bar{G}^{-1}<0$ if $u<0$. 
Lemma 2. Define coordinates and time transformation $T_{2}$ as

$$
T_{2}: u=\sqrt{2 \bar{G}(x)} \operatorname{sgn} x, \quad y=y, \quad \frac{d s}{d t}=\frac{a(x)|g(x)|}{\sqrt{2 \bar{G}(x)}},
$$

where $\bar{G}(x)=\int_{0}^{x} a^{2}(s) g(s) d s$. Then under the transformation $T_{2}$, system (2) is changed into the following form:

$$
\left\{\begin{array}{l}
\dot{u}=h(y)-\bar{F}^{*}(u), \\
\dot{y}=-u,
\end{array}\right.
$$

where

$$
\bar{F}^{*}(u)=F\left(\bar{G}^{-1}\left(u^{2} / 2\right)\right)
$$

with $\bar{G}^{-1}>0$ if $u>0$ and $\bar{G}^{-1}<0$ if $u<0$.

Lemma 3. If $A, B$ are nonnegative numbers and $p>1, q=\frac{p}{p-1}$, then

$$
\frac{A^{p}}{p}+\frac{B^{q}}{q} \geq A B
$$

where the equality holds if and only if $A=B^{q-1}$.

The proofs of Lemma $1 \sim$ Lemma 3 are straightforward, so we omit them.

\section{Main Results And Proofs}

Now, we can state the main results of this paper. For simplicity, we discuss only the property $X^{+}$in the right half-plane.

Theorem 1. Define a positive constant $\lambda_{p}$ by

$$
\lambda_{p}=\frac{p}{(p-1)^{\frac{2(p-1)}{p}}} .
$$

Suppose that for some $b>0$, we have

$$
\limsup _{u \rightarrow+\infty}\left(\int_{b}^{u} \frac{F^{*}(s)+\lambda_{p} s^{p-1}}{s^{p}} d s+\frac{F^{*}(u)}{(p-1) u^{p-1}}\right)=+\infty,
$$

where $F^{*}$ is defined in Lemma 1. Then system (1) has property $X^{+}$in the right half-plane.

Remark. Note that if $p=2$, then Theorem 1 reduces to Theorem A in [12]. Since $u=(p \bar{G}(x))^{1 / p}$ for $x \geq 0$, setting $s=(p \bar{G}(t))^{1 / p}$ and $b=(p \bar{G}(c))^{1 / p}$ in (9), we can obtain the following corollary: 
Corollary 1. Assume that $\bar{G}(+\infty)=+\infty$ and that for some $c>0$, we have

$$
\limsup _{x \rightarrow+\infty}\left[\int_{c}^{x}\left(\frac{F(t) a^{q}(t) g(t)}{(p \bar{G}(t))^{\frac{2 p-1}{p}}}+\frac{\lambda_{p} a^{q}(t) g(t)}{p \bar{G}(t)}\right) d t+\frac{F(x)}{(p-1)(p \bar{G}(x))^{\frac{p-1}{p}}}\right]=+\infty .
$$

Then system (1) has property $X^{+}$in the right half-plane.

Remark. Note that if $a(x)=1, p=2$, then Corollary 1 reduces to Theorem B in [12].

Theorem 2. Let $\mu=2 \sqrt{m}$ and suppose that for some $b>0$, we have

$$
\limsup _{u \rightarrow+\infty}\left(\int_{b}^{u} \frac{\bar{F}^{*}(s)+\mu s}{s^{k+1}} d s+\frac{\bar{F}^{*}(u)}{k u^{k}}\right)=+\infty,
$$

where $\bar{F}^{*}$ is defined in Lemma 2. Then system (2) has property $X^{+}$in the right half-plane.

Since $u=(2 \bar{G}(x))^{1 / 2}$ for $x \geq 0$, setting $s=(2 \bar{G}(t))^{1 / 2}$ and $b=(2 \bar{G}(d))^{1 / 2}$ in (11), we can obtain the following corollary:

Corollary 2. Assume that $\bar{G}(+\infty)=+\infty$ and that for some $d>0$,

$$
\limsup _{x \rightarrow+\infty}\left[\int_{d}^{x}\left(\frac{F(t) a^{2}(t) g(t)}{(2 \bar{G}(t))^{\frac{k+2}{2}}}+\frac{\mu a^{2}(t) g(t)}{(2 \bar{G}(t))^{\frac{k+1}{2}}}\right) d t+\frac{F(x)}{k(2 \bar{G}(x))^{\frac{k}{2}}}\right]=+\infty .
$$

Then system (2) has property $X^{+}$in the right half-plane.

Proof of Theorem 1. By Lemma 1, we need only to consider system (7). We still denote $s$ by $t$ for simplicity. We prove the theorem by contradiction. Suppose that there exists a solution $(u(t), y(t))$ of system (7) whose orbit remains in the region $D=\left\{(u, y): u \geq 0\right.$, and $\left.y>F^{*}(u)\right\}$ for all $t>0$, which implies that $\dot{u}(t)>0 \forall t>0$. Let $\left(u_{0}, y_{0}\right)=(u(0), y(0))$. Since system (7) has no critical points in this region $D$, we have

$$
\lim _{t \rightarrow+\infty} u(t)=+\infty
$$

and so we may assume that $u_{0}>0$ is sufficiently large. We may also assume that $y_{0} \leq 0$ since

$$
y(t) \leq y_{0}-u_{0}^{p-1} t \rightarrow-\infty, \quad \text { as } t \rightarrow+\infty .
$$

Therefore, we may assume that

$$
u(t) \geq u_{0}>0 \quad \text { and } \quad y(t) \leq y_{0} \leq 0 \quad \text { for } t \geq 0 .
$$


Define a function $L(t)$ by

$$
L(t)=\int_{b}^{u(t)} \frac{F^{*}(s)}{s^{p}} d s+\frac{y(t)}{(p-1) u^{p-1}(t)} .
$$

By using (7) and Lemma 3, we obtain

$$
\begin{aligned}
\frac{d}{d t} L(t) & =\frac{F^{*}(u(t)) \dot{u}(t)}{u^{p}(t)}-\frac{(p-1) u^{p-2}(t) \dot{u}(t) y(t)-u^{p-1}(t) \dot{y}(t)}{(p-1) u^{2(p-1)}(t)} \\
& =\frac{\left(y(t)-\phi_{p}(\dot{u}(t))\right) \dot{u}(t)}{u^{p}(t)}-\frac{y(t) \dot{u}(t)}{u^{p}(t)}-\frac{1}{p-1} \\
& =-\frac{(p-1)(\dot{u}(t))^{p}+u^{p}(t)}{(p-1) u^{p}(t)} \\
& \leq-\frac{p \dot{u}(t) u^{p-1}(t)}{(p-1)^{\frac{2(p-1)}{p}} u^{p}(t)}=-\frac{p \dot{u}(t)}{(p-1)^{\frac{2(p-1)}{p}} u(t)}=-\frac{\lambda_{p} \dot{u}(t)}{u(t)},
\end{aligned}
$$

where we set

$$
A^{p}=p(p-1)(\dot{u}(t))^{p}, \quad B^{q}=q u^{p}(t)
$$

in Lemma 3. This implies that

$$
\frac{d}{d t}\left(L(t)+\int_{b}^{u(t)} \frac{\lambda_{p} d s}{s}\right) \leq 0
$$

which again implies that

$$
\frac{d}{d t}\left(\int_{b}^{u(t)} \frac{F^{*}(s)+\lambda_{p} s^{p-1}}{s^{p}} d s+\frac{y(t)}{(p-1) u^{p-1}(t)}\right) \leq 0 .
$$

We have therefore

$$
\begin{aligned}
& \int_{b}^{u(t)} \frac{F^{*}(s)+\lambda_{p} s^{p-1}}{s^{p}} d s+\frac{y(t)}{(p-1) u^{p-1}(t)} \\
& \quad \leq \int_{b}^{u_{0}} \frac{F^{*}(s)+\lambda_{p} s^{p-1}}{s^{p}} d s+\frac{y_{0}}{(p-1) u_{0}^{p-1}}<+\infty \quad \text { for } t \geq 0 .
\end{aligned}
$$

Since $y(t)>F^{*}(u(t))$ by the assumption and $u(t) \rightarrow+\infty$ as $t \rightarrow+\infty$, we obtain

$$
\limsup _{u \rightarrow+\infty}\left(\int_{b}^{u} \frac{F^{*}(s)+\lambda_{p} s^{p-1}}{s^{p}} d s+\frac{F^{*}(u)}{(p-1) u^{p-1}}\right)<+\infty,
$$

which contradicts the assumption of Theorem 1 .

Proof of Theorem 2. By Lemma 2, we need only to consider system (8). We again denote $s$ by $t$ and prove the theorem by contradiction. Suppose that there exists an orbit $(u(t), y(t))$ of system (8) which remains in the region $\bar{D}=\{(u, y): u \geq$ 
0 and $\left.h(y)>\bar{F}^{*}(u)\right\}$ for all $t>0$. Like the analysis in the proof of Theorem 1 , we may assume that

$$
u(t) \geq u_{0}>0, \quad \text { and } \quad y(t) \leq y_{0} \leq-1 \quad \text { for } t \geq 0 .
$$

For $b>0$ fixed, define a function $H(t)$ by

$$
H(t)=\int_{b}^{u(t)} \frac{\bar{F}^{*}(s)}{s^{k+1}} d s+\frac{h(y(t))}{k u^{k}(t)} .
$$

Since $y(t) \leq-1$ and $k \geq 1$, we have $|y(t)| \geq 1$ and $h^{\prime}(y(t))=m k|y(t)|^{k-1} \geq m k$. Now using (8) and Lemma 3 with $p=q=2$, we have

$$
\begin{aligned}
\frac{d}{d t} H(t) & =\frac{\bar{F}^{*}(u(t)) \dot{u}(t)}{u^{k+1}(t)}+\frac{u^{k}(t) h^{\prime}(y(t)) \dot{y}(t)-k u^{k-1}(t) h(y(t)) \dot{u}(t)}{k u^{2 k}(t)} \\
& =-\frac{k \dot{u}^{2}(t)+h^{\prime}(y(t)) u^{2}(t)}{k u^{k+1}(t)} \leq-\frac{k \dot{u}^{2}(t)+m k u^{2}(t)}{k u^{k+1}(t)} \\
& =-\frac{\dot{u}^{2}(t)+m u^{2}(t)}{u^{k+1}(t)} \leq-\frac{2 \sqrt{m} \dot{u}(t) u(t)}{u^{k+1}(t)}=-\frac{\mu \dot{u}(t)}{u^{k}(t)},
\end{aligned}
$$

which implies

$$
\frac{d}{d t}\left(H(t)+\int_{b}^{u(t)} \frac{\mu}{s^{k}} d s\right) \leq 0 .
$$

Hence, for $t \geq 0$, we obtain

$$
\int_{b}^{u(t)} \frac{\bar{F}^{*}(s)+\mu s}{s^{k+1}} d s+\frac{h(y(t))}{k u^{k}(t)} \leq \int_{b}^{u_{0}} \frac{\bar{F}^{*}(s)+\mu s}{s^{k+1}} d s+\frac{h\left(y_{0}\right)}{k u_{0}^{k}}<+\infty .
$$

Since $h(y(t))>\bar{F}^{*}(u(t))$ and $u(t) \rightarrow+\infty$ as $t \rightarrow+\infty$, we get

$$
\limsup _{u \rightarrow+\infty}\left(\int_{b}^{u} \frac{\bar{F}^{*}(s)+\mu s}{s^{k+1}} d s+\frac{\bar{F}^{*}(u)}{k u^{k}}\right)<+\infty .
$$

This contradicts the assumption of Theorem 2 .

\section{ExAmples}

Example 1. Let us consider system (1). Let $a^{q}(x)=\frac{1}{1+x^{2}}, g(x)=2 x$. Then $\bar{G}(x)=\ln \left(1+x^{2}\right)$ and $\bar{G}(+\infty)=+\infty$. Assume that for $x \geq 0$ and some $\delta \in[0,1)$, we have $F(x) \geq-\delta \lambda_{p}(p \bar{G}(x))^{\frac{p-1}{p}}=-\delta \lambda_{p}\left(p \ln \left(1+x^{2}\right)\right)^{\frac{p-1}{p}}$. Then we get for $u \geq 0$,

$$
F^{*}(u) \geq-\delta \lambda_{p} u^{p-1} \text { and } \quad \frac{F^{*}(u)}{(p-1) u^{p-1}} \geq-\frac{\delta \lambda_{p}}{(p-1)} .
$$


This yields

$$
\begin{aligned}
\int_{b}^{u} \frac{F^{*}(s)+\lambda_{p} s^{p-1}}{s^{p}} d s+\frac{F^{*}(u)}{(p-1) u^{p-1}} & \geq \int_{b}^{u} \frac{(1-\delta) \lambda_{p}}{s} d s-\frac{\delta \lambda_{p}}{p-1} \\
& =(1-\delta) \lambda_{p} \ln \frac{u}{b}-\frac{\delta \lambda_{p}}{p-1} .
\end{aligned}
$$

Hence we have

$$
\limsup _{u \rightarrow+\infty}\left[\int_{b}^{u} \frac{F^{*}(s)+\lambda_{p} s^{p-1}}{s^{p}} d s+\frac{F^{*}(u)}{(p-1) u^{p-1}}\right]=+\infty .
$$

Therefore, by Theorem 1, system (1) has property $X^{+}$in the right half-plane.

Example 2. Let us consider system (2). First, if $k=1$, assume that for $x \geq 0$ and some $\delta \in[0,1), F(x) \geq-2 \delta \sqrt{2 m \bar{G}(x)}$. Then we have for $u \geq 0$,

$$
\begin{aligned}
\int_{b}^{u} \frac{\bar{F}^{*}(s)+2 \sqrt{m} s}{s^{2}} d s+\frac{\bar{F}^{*}(u)}{u} & \geq \int_{b}^{u} \frac{2(1-\delta) \sqrt{m}}{s} d s-2 \delta \sqrt{m} \\
& =2(1-\delta) \sqrt{m} \ln \left(\frac{u}{b}\right)-2 \delta \sqrt{m}
\end{aligned}
$$

which implies

$$
\limsup _{u \rightarrow+\infty}\left[\int_{b}^{u} \frac{\bar{F}^{*}(s)+2 \sqrt{m} s}{s^{2}} d s+\frac{\bar{F}^{*}(u)}{u}\right]=+\infty .
$$

Second, if $k>1$, we assume that for $x \geq 0$ and some $\delta \in[0,1)$,

$$
F(x) \geq-2 \delta \sqrt{2 m \bar{G}(x)}
$$

and that $\bar{G}(+\infty)=+\infty$ and $\lim \sup _{x \rightarrow+\infty} F(x) /(2 \bar{G}(x))^{k / 2}=+\infty$. Then this implies for $u \geq 0$,

$$
\bar{F}^{*}(u) \geq-2 \delta \sqrt{m} u \text { and } \quad \limsup _{u \rightarrow+\infty} \frac{\bar{F}^{*}(u)}{u^{k}}=+\infty .
$$

In this case, we have

$$
\limsup _{u \rightarrow+\infty}\left[\int_{b}^{u} \frac{\bar{F}^{*}(s)+2 \sqrt{m} s}{s^{k+1}} d s+\frac{\bar{F}^{*}(u)}{k u^{k}}\right] \geq \limsup _{u \rightarrow+\infty} \frac{\bar{F}^{*}(u)}{k u^{k}}=+\infty .
$$

Hence, by Theorem 2, system (2) has property $X^{+}$in the right half-plane.

\section{REFERENCES}

1. J.G. Wendel: On a Van Der Pol equation with odd coefficients. J. London Math. Soc. 24 (1949), 65-67.

2. A.F. Filippov: A sufficient condition for the existence of a stable limit cycle of second order equation. Mat. Sb. (1952) 30, no. 72, 171-180. 
3. Z. Opial: Sur un theoreme de A.Filippoff. Ann. Polon. Math. 5 (1958), 67-75.

4. V.V. Nemyckii \& V.V. Stepanov: Qualitative Theory of Ordinary Differential Equations. English ed, Princeton Univ. Press, Princeton, NJ, 1960.

5. T. Hara \& T. Yoneyama: On the global center of generalized Liénard equation and its applications to stability problems. Funkcial Ekvac. 28 (1985), 171-192.

6. G. Villari \& F. Zanolin: On a dynamical system in the Liénard plane. Necessary and sufficient conditions for the intersection with the vertical isocline and applications. Funkcial Ekvac. 33 (1990), 19-38.

7. J. Sugie: The global center for the Liénard system. Nonlinear Analysis 17(1991), 333345 .

8. S. Yu: On the center of Liénard system. J. Diff. Equa. 102 (1993), 53-61.

9. J. Sugie \& T. Hara: Classification of global phase portraits of a system of Liénard type. J. Math. Anal. Appl. 193 (1995), 264-281.

10. J. Sugie, D.L. Chen \& H. Matsunaga: On global asymptotic stability of systems of Liénard type. J. Math. Anal. Appl. 219 (1998), 140-164.

11. J. Sugie: Homoclinic orbits in generalized Liénard systems. J. Math. Anal. Appl. 309 (2005), 211-226.

12. A. Aghajani \& A. Moradifam: Some sufficient conditions for the intersection with the vertical isocline in the Liénard plane. Appl. Math. Letters 19 (2006), 491-497.

13. M. Hesaaraki \& A. Moradifam: Intersection with the vertical isocline in the generalized Liénard equations. J. Math. Anal. Appl. 334 (2007), 787-795.

14. A. Aghajani \& A. Moradifam: On the homoclinic orbits of the generalized Liénard equations. Appl. Math. Letters. 20 (2007), 345-351.

15. J. Sugie, A. Kono \& A. Yamaguchi: Existence of limit cycles for Liénard-type systems with p-Laplacian. Nonlinear Differ. Equ. Appl. 14 (2007), 91-110.

Department of Mathematics, University of Ulsan, Ulsan 689-749, Korea

Email address: yikim@mail.ulsan.ac.kr 